\title{
Sistema de recuperación de energía basado en emular el movimiento del flujo de agua en tuberías
}

\section{Energy recovery system based on emulating the movement of water flow in pipes}

\author{
MASTACHE-MASTACHE，Jorge Edmundo†**, RAMÍREZ-CHÁVEZ，César León， LOVERA- \\ GARCIA, Jesús Adan y DE LA GARZA-MACEDO, José Ángel
}

Universidad de Ixtlahuaca CUI, Escuela Profesional de Ingeniería y TESJO

ID $1^{\mathrm{er}}$ Autor: Jorge Edmundo, Mastache-Mastache / ORC ID: 0000-0001-6104-6764, Researcher ID Thomson: H-11872018, CVU CONACYT ID: 544943

ID 1 ${ }^{\mathrm{er}}$ Coautor: César León, Ramírez-Chávez / ORC ID: 0000-0002-3603-6422, CVU CONACYT ID: 163468

ID $2^{\text {do }}$ Coautor: Jesús Adan, Lovera-Garcia / ORC ID: 0000-0002-1145-103X

ID $3^{\text {er }}$ Coautor: José Ángel, De La Garza-Macedo / ORC ID: 0000-0002-7744-3307

DOI: $10.35429 / J T E N .2020 .13 .4 .1 .8$

Recibido 03 de Marzo, 2020; Aceptado 30 Junio, 2020

\begin{abstract}
Resumen
La producción de energía es un factor importante para el desarrollo de actividades productivas, de transformación y servicios en el país, cuya generación y consumo incrementa año con año, lo que conlleva a utilizar cada vez más las energías limpias. La energía hidroeléctrica como opción portátil de aplicación aporta poco para la producción energética en industrias u hogares, por lo que esta situación presenta una oportunidad para el desarrollo de sistemas adaptables de generación de energía que aprovechan el movimiento del agua. En el presente trabajo se desarrolló un banco de pruebas basado en un sistema de recuperación de energía, el cual consiste en simular el flujo de agua que existe en las tuberías para así generar energía eléctrica a través del movimiento mecánico acoplando un generador de imanes permanentes, donde se emuló el comportamiento de una turbina interconectada a una tubería, a través de un motor de CD cuya velocidad es controlada a través de un troceador de potencia. Se desarrolló un almacenamiento y conversión de potencia, integrando un módulo rectificador con filtro el cual convertía la señal de alterna a directa, que posteriormente seria la entrada de un convertidor CD-CD reductor Buck, el cual tiene una alta eficiencia y permite reducir el voltaje estabilizado a 5V. Para completar el sistema de almacenamiento a diferentes cargas se utilizó un módulo convertidor de potencia Boost el cual eleva y convierte la señal de CD a CD, obteniendo una salida de tensión mayor que su entrada, esta energía podrá ser almacenada en baterías y de igual forma ser utilizado para energizar cualquier dispositivo de $\mathrm{CD}$, permitiéndonos así ahorrar una parte de la energía que se consume al transportar el agua en una tubería.
\end{abstract}

Energía hidráulica, Banco de Pruebas, Convertidores

\begin{abstract}
Energy production is an important factor for the development of productive activities, transformation and services in the country, whose generation and consumption increases year by year, which leads to the use of clean energy more and more. Hydropower as a portable application option contributes little to energy production in industries or homes, so this situation presents an opportunity for the development of adaptive power generation systems that take advantage of the movement of water. In the present work, a test bench based on an energy recovery system was developed, which consists of simulating the flow of water that exists in the pipes in order to generate electrical energy through mechanical movement by coupling a permanent magnet generator, where the behavior of a turbine interconnected to a pipeline was emulated, through a DC motor whose speed is controlled through a power chopper. A storage and power conversion was developed, integrating a rectifier module with filter which converted the signal from alternating to direct, which would later be the input of a Buck reducing CD-CD converter, which has high efficiency and allows to reduce the voltage stabilized at $5 \mathrm{~V}$. To complete the storage system at different loads, a Boost power converter module was used which raises and converts the signal from $\mathrm{CD}$ to $\mathrm{CD}$, obtaining a voltage output greater than its input, this energy can be stored in batteries and in the same way be used to power any $\mathrm{CD}$ device, thus allowing us to save a part of the energy that is consumed when transporting water in a pipe.
\end{abstract}

Hydraulic energy, Test Bench, Converters.

Citación: MASTACHE-MASTACHE, Jorge Edmundo, RAMÍREZ-CHÁVEZ, César León, LOVERA-GARCIA, Jesús Adan y DE LA GARZA-MACEDO, José Ângel. Sistema de recuperación de energía basado en emular el movimiento del flujo de agua en tuberías. Revista de Ingeniería Tecnológica. 2020. 4-13: 1-8

\footnotetext{
* Correspondencia del Autor (mastache@uicui.edu.mx)

$\dagger$ Investigador contribuyendo como primer autor.
} 


\section{Introducción}

En la última década, la industria eléctrica registró tasas de crecimiento positivas, siendo la segunda fuente de energía con mayor consumo en México, con una participación de 30.4\% de consumo energético total del país. (SENER 2018) Representando el $23.5 \%$ del consumo de energía en el sector agropecuario, el $36.1 \%$ en la industrial el $30.1 \%$ del consumo total en los sectores residencial, comercial y público y el $0.2 \%$ del sector transporte. La Figura 1 muestra la gráfica del consumo de energía en cada sector.

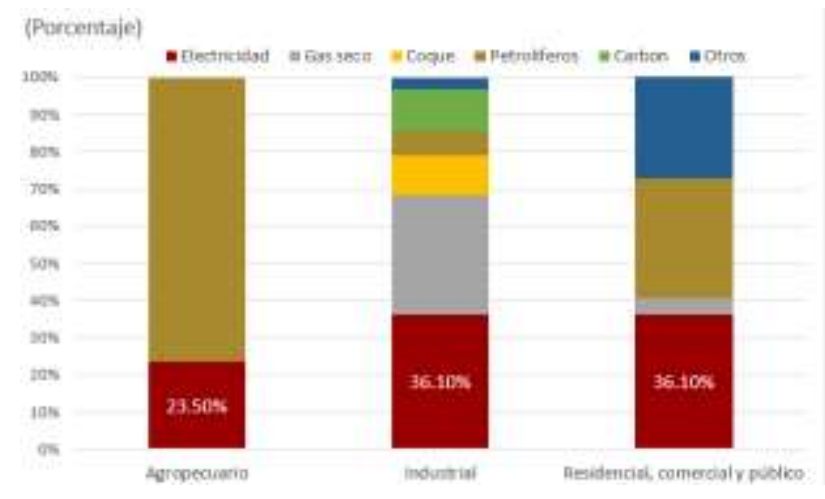

Figura 1 Participación de la electricidad en el consumo final de energía por sector (porcentaje)

Fuente: SENER, (2018)

La energía eléctrica representa el segundo gasto de consumo total en las viviendas, junto con el agua, gas y otros combustibles según la Encuesta Nacional de los Hogares realizada por el INEGI. Por hogar se entiende el conjunto de personas que pueden ser o no familiares, que comparten la misma vivienda y se sostienen de un gasto común. (INEGI 2018) La demanda de energía eléctrica representa un gasto en los hogares mexicanos, el cual es el segundo gasto de consumo total de las viviendas, estimando un gasto promedio trimestral en la electricidad es de $\$ 1,388$ pesos, por hogar.

Dichas cifras según las encuestas van aumentando de acuerdo a las personas que viven en los hogares, puesto que utilizan la energía para actividades cotidianas y por lo tanto los usuarios destinan una mayor parte de su ingreso trimestral hacia al pago de la electricidad. (SENER 2018) México está dominado por las fuentes no renovables de energía, las cuales han jugado un papel decisivo para el desarrollo económico del país, sin embargo, aunque consumir este tipo de energía es necesario para los proceso productivos de la sociedad, debemos considerar que producen un daño considerable al medio ambiente.
En los últimos años la búsqueda de nuevas fuentes de energía, ha desarrollado estudios de sistemas o dispositivos que sean eficientes $\mathrm{o}$ propongan nuevas formas de recuperación o almacenamiento de energía, es por esto que la comunidad científica se decide investigar sobre las fuentes de Energía Renovables. Una de estas fuentes es la energía hidroeléctrica, además de ser accesible para casi todos los países se puede aprovechar para casi todos los cursos o vías de agua.

En otros términos, significa aprovechar una fuerza que se produce naturalmente. Una instalación hidráulica se puede definir como el conjunto de tubos, muebles, accesorios (válvulas, codos y conexiones) y equipo (calentadores, bombas, hidroneumáticos) unidos para llevar en forma adecuada el suministro de agua fría y/o caliente a una edificación. Es por esto que en este trabajo se propone desarrollar un banco de pruebas basado en el diseño e implementación de un sistema electromecánico, capaz de recuperar energía a través del movimiento de agua en las tuberías, el cual servirá como punto de partida para desarrollar estudios de electrónica de potencia, recuperación de energía y control de sistemas dinámicos, pretendiendo que el sistema pueda ser instalado posteriormente en las casas habitación, cuya energía será generada a través del flujo del agua que desciende de las tuberías, hacia el sistema mecánico que estará compuesto por una turbina conectada a un generador de imanes permanentes, el cual producirá una señal eléctrica generada de $\mathrm{CA}$, que deberá ser acoplada a través de convertidores de potencia para su posterior utilización o almacenamiento en baterías.

El presente trabajo servirá como base para futuros proyectos con el fin de ahorrar una parte de la energía que es consumida diariamente en los hogares, ya que toda estructura arquitectónica cuenta con una distribución de agua potable a través de tuberías, la cual se mantiene en constante movimiento por las actividades cotidianas que se realizan como lavarse las manos, bañarse, lavar la ropa, etc., éste movimiento no es aprovechado, por lo que se pretende recuperar esta energía a través de un sistema electromecánico, donde estaremos generando energía sin ningún tipo de daño al medio ambiente. 


\section{Metodología}

Es necesario identificar los elementos del prototipo de banco de pruebas del sistema electromecánico de recuperación de energía, para ello se propone el diagrama mostrado en la figura 2.

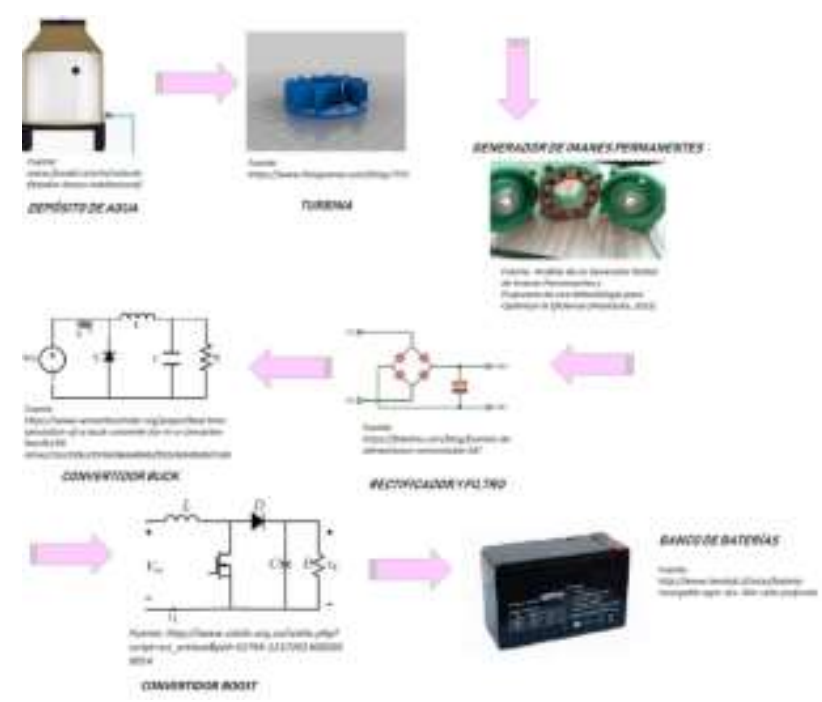

Figura 1 Diagrama representante del sistema Fuente: Elaboración Propia

El sistema consta de la emulación de una turbina instalada directamente en la tubería de agua potable que estará conectada directamente hacia el tinaco, dicha turbina estaría acoplada por una flecha hacia un generador de imanes permanentes, cuya energía generada se traslada hacia un circuito de rectificación y filtrado. Posteriormente, el voltaje obtenido se conducirá hacia un convertidor CD-CD Buck, el cual disminuirá la tensión a $5 \mathrm{~V}$ y después se incluye un convertidor CD-CD para elevar la tensión a 12-24 V, lo que permitirá utilizar para diferentes tipos de cargas o su almacenamiento en baterías.

\section{Diseño del banco de pruebas}

Como se presentó en el diagrama anterior el banco de pruebas, se subdivido en diferentes etapas de diseño, las cuales se describen de forma individual y se presentan a continuación. Dichas etapas son:

\footnotetext{
- $\quad$ Análisis y acoplamiento mecánico

- $\quad$ Sistema de control de velocidad

- $\quad$ Sistema de recuperación de energía
}

\section{Análisis y acoplamiento mecánico}

Las instalaciones hidráulicas tienen la función de abastecer de agua los diferentes tipos de edificaciones y dentro de estos, está proviene convencionalmente de fuentes de almacenamiento a través de tinacos o depósitos de agua, los cuales emplean el sistema de tanque elevado, por gravedad, o de tanque a presión. Dichos tinacos se encuentran instalados en la parte superior de las construcciones, cuya conexión desciende hacia los muebles por tuberías, generalmente de cobre, que es la más utilizada para casas habitación por su alta resistencia a temperaturas altas y cuya medida estándar es de 19 mm de diámetro.

En estas tuberías existe energía potencial y cinética que no es aprovechada, por lo tanto, este movimiento puede ser aprovechado para hacer girar una turbina. Se calculó la velocidad del flujo de agua en las tuberías y para esto fue necesario comprobar el caudal que atraviesa dicha tubería, para ello se hace una estimación de la velocidad del agua que fluye a través del orificio del tinaco de acuerdo al Teorema de Torricelli, con lo cual se concluye que la velocidad en las tuberías de agua que descienden del tinaco es de $5.03 \mathrm{~m} / \mathrm{s}$. Por lo tanto, se propone el diseño de una turbina Pelton puesto que son ideales para las aplicaciones con caudales pequeños.

Para la simulación de esta turbina se propuso desarrollar un acoplamiento motorgenerador, los cuales controlaremos con una etapa de control de velocidad electrónico, para emular el comportamiento del flujo de agua en tuberías, determinando las revoluciones por minuto (R.P.M.) a partir de la velocidad y el diámetro del rotor asociado a la construcción física, el cual debe girar a 434 R.P.M. Este sistema consta de un motor de $18 \mathrm{~V}$ acoplado hacia el generador de imanes permanentes a través de una flecha de fierro, así mismo se integran 2 chumaceras para brindar apoyo al eje de rotación del motor.

La etapa de acoplamiento mecánico puede mostrarse en el dibujo diseñado en un paquete de dibujo CAD, para representar el acoplamiento mecánico en el diseño del banco de pruebas como se muestra en la Figura 3. 


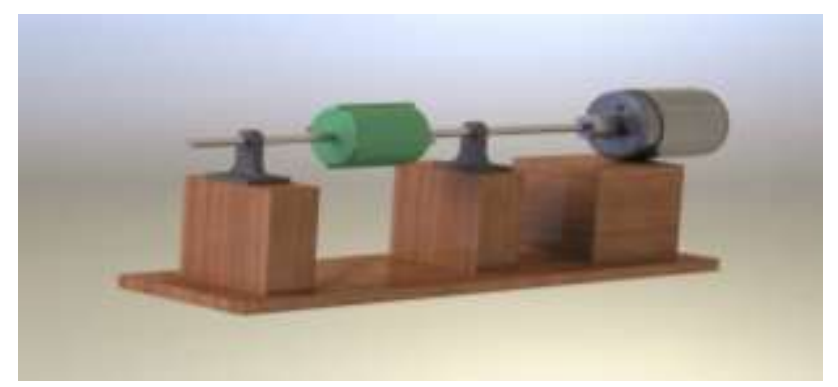

Figura 3 Diseño del banco de pruebas en software CAD Fuente: Elaboración Propia

\section{Sistema de control de velocidad}

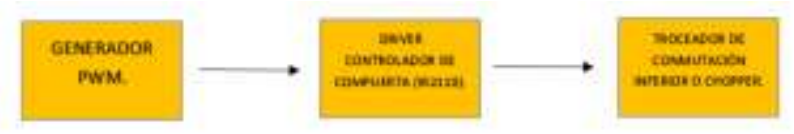

Figura 1 Diagrama a bloques del control de velocidad Fuente: Elaboración Propia

Como se muestra en el diagrama a bloques para emular el comportamiento de la turbina que requiere el sistema, se acoplo el generador a un motor de CD a $18 \mathrm{~V}$ a $4.1 \mathrm{~A}$ cuya velocidad es controlada a través de un circuito electrónico troceador conmutación inferior, controlado bajo la técnica de modulación PWM por el circuito TL494 y acoplado a través de un driver de controlador de compuerta IR2110, para provocar la conmutación en el MOSFET semiconductor para el switcheo de una fuente, emulando así a través de la potencia reflejada a la carga las R.P.M. que genera la turbina con el flujo de agua de una tubería, como se muestra en la simulación de la figura siguiente desarrollada en el software PROTEUS 8.9.

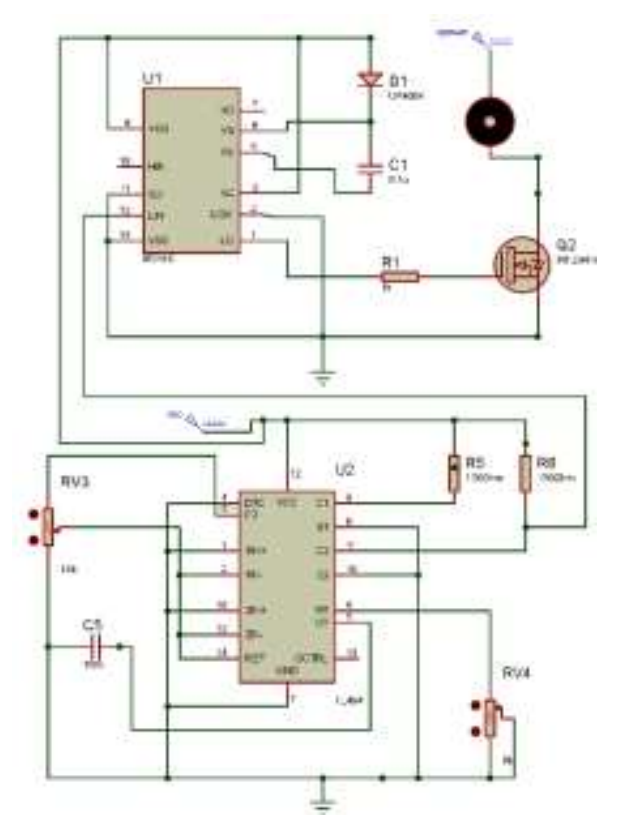

Figura 5 Diagrama esquemático del control de velocidad del motor

Fuente: Elaboración Propia

\section{Sistema de recuperación de energía}

En el diagrama siguiente se muestra la división de los circuitos que se tuvieron que desarrollar para lograr el prototipo de sistema de recuperación.

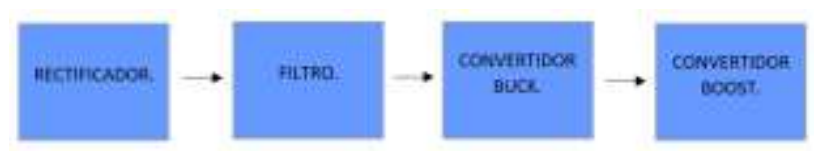

Figura 6 Diagrama de bloques del circuito de recuperación de energía

Fuente: Elaboración Propia

Para diseñar el circuito de recuperación de energía se parte, de emular la señal a la salida del generador de imanes permanentes en un software de simulación de circuitos eléctricos PSIM 9.0, la cual fue representada a través de una fuente de CA como se muestra en la figura 7 , esta señal es dirigida a un módulo rectificador de onda completa conformado por 4 diodos de germanio para aprovechar el bajo voltaje umbral de los dispositivos y reducir las pérdidas de conducción en esta etapa, la salida del rectificador fue filtrada y acoplada a la siguiente etapa.

A la salida del rectificador CA a CD se añadió un módulo convertidor de potencia Buck, el cual es un convertidor reductor el cual presenta la propiedad de siempre tener un voltaje menor a la salida en comparación con la entrada, esto debido al switcheo del transistor el cual en el instante $t=0$ comienza a conducir el interruptor semiconductor de potencia, formando dos subcircuitos equivalente de esta etapa donde Vo es menor que Vin y la corriente que circula por el inductor L será creciente durante el intervalo del tiempo de encendido.

Posteriormente en el tiempo apagado se genera entonces una sobretensión que hace conducir al diodo en polarización directa generando un nuevo subcircuito que provoca que la energía almacenada en el inductor se reste con el voltaje de alimentación del convertidor, esto con la finalidad de estabilizar la señal de salida del convertidor, debido a que la señal de entrada es cambiante tanto en amplitud como en frecuencia según el flujo de agua en las tuberías. 
Después se integró un módulo convertidor de potencia Boost el cual es un elevador de tensión que consiste en convertir energía CD a CD, obteniendo una salida de tensión continua mayor a su entrada, es un tipo de fuente de alimentación conmutada que contiene dos interruptores semiconductores (diodo y transistor); y un elemento inductivo para almacenar energía, que del mismo modo al pasar a un estado de encendido el interruptor de potencia, carga un inductor el tiempo definido por el ciclo de trabajo y en el tempo de apagado este se suma al nivel de referencia de entrada provocando la elevación de voltaje a la salida del convertidor y de esta forma como se ha mencionado alimentar diferentes tipos de cargas y almacenamiento en baterías mayores a 5V. En la figura 7 se muestra el circuito esquemático en el software PSIM 9.0 completo del sistema de recuperación de energía que fue acoplado al sistema mecánico propuesto en las secciones anteriores.

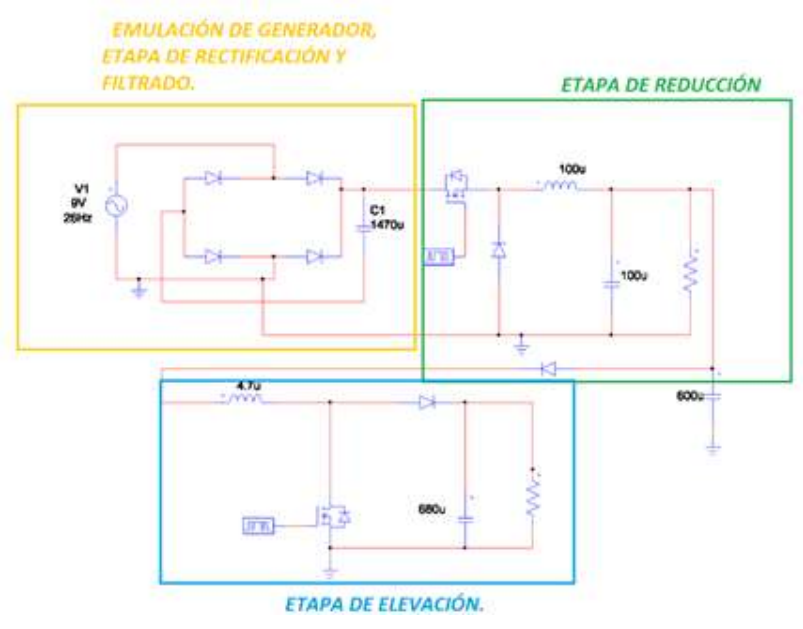

Figura 7 Diagrama esquemático del sistema de recuperación de energía Fuente: Elaboración Propia

\section{Resultados}

\section{Acoplamiento mecánico}

En la Figura 8 se muestra el armado físico del sistema mecánico del banco del pruebas constituido por motor regulado por el control de velocidad, conectado a su vez al generador de imanes permanentes a través de una flecha de fierro como acople mecánico, para emular el comportamiento de la turbina. La base anteriormente diseñada, está construida de madera para brindar mayor estabilidad por la vibración del movimiento del motor.

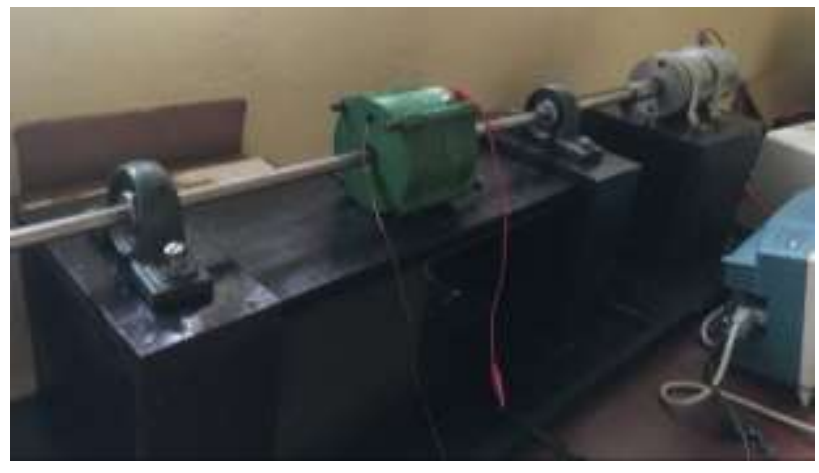

Figura 8 Implementación de estructura mecánica del banco de pruebas

Fuente: Elaboración Propia

La turbina del sistema fue emulada por un motor de CD de $18 \mathrm{~V}$, el cual es controlado por circuito de control de velocidad, simulando así la velocidad con la que desciende el agua en las tuberías de agua potable. El motor se muestra en la Figura 9.

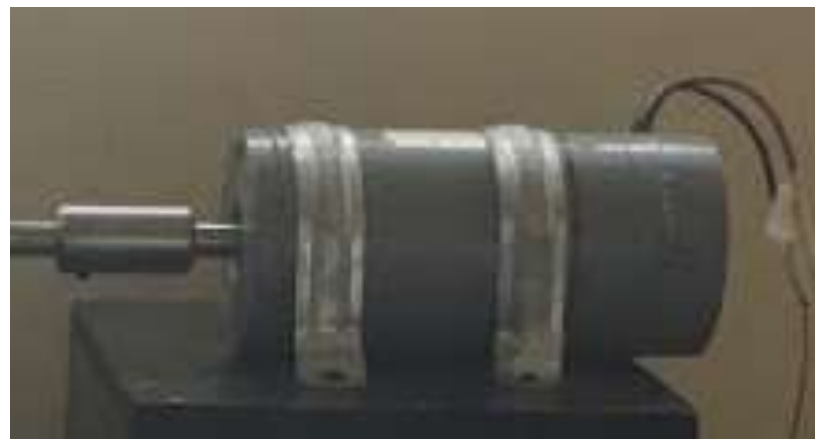

Figura 9 Motor de CD

Fuente: Elaboración Propia

\section{Generador de imanes permanentes}

Se utiliza un generador de imanes de 10 polos diseñado e implementado en trabajos previos del grupo de investigación en la tesis de Maestría "Análisis de un Generador Radial de Imanes Permanentes y Propuesta de una Metodología para Optimizar la Eficiencia" (Mastache 2016) el cual se muestra en la Figura 10, que tiene un rotor de fierro en forma de decágono en donde se adhieren 10 imanes permanentes de neodimio de $40 \mathrm{~mm}$ de largo por $15 \mathrm{~mm}$ ancho por $2 \mathrm{~mm}$ de grosor, el rotor y el imán se muestran en la Figura 10 respectivamente.

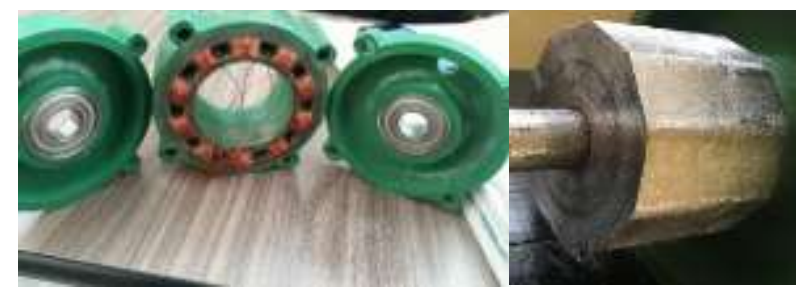

Figura 10 Generador de imanes permanentes de 10 polos Fuente: Elaboración Propia

MASTACHE-MASTACHE, Jorge Edmundo, RAMÍREZ-CHÁVEZ, César León, LOVERA-GARCIA, Jesús Adan y DE LA GARZAMACEDO, José Ángel. Sistema de recuperación de energía basado en emular el movimiento del flujo de agua en tuberías. Revista de Ingeniería Tecnológica. 2020 
En la Figura 11 se muestra la captura de pantalla del osciloscopio Tektronik TBS-1202BEDU, la cual es señal de salida del generador de imanes permanentes con el motor controlado a 434 r.p.m. medidas, con las que se pudieron ajustar a partir del circuito para el control de velocidad, obteniendo una amplitud de voltaje máximo de $10 \mathrm{~V}$.

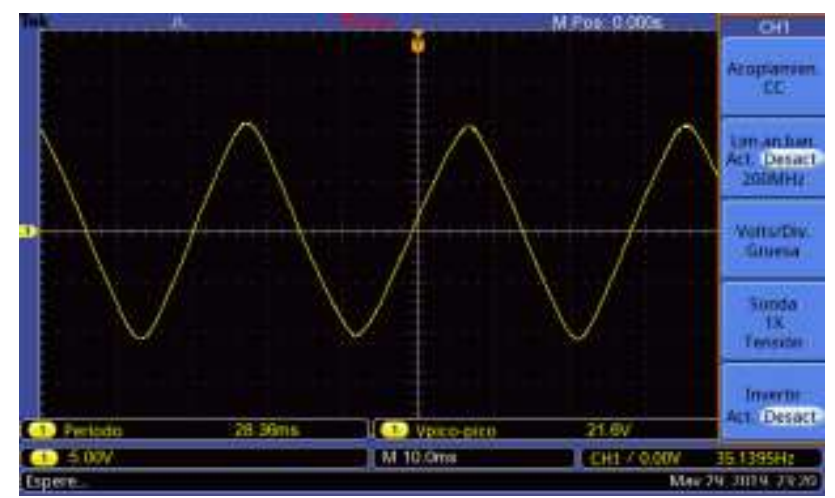

Figura 11 Señal senoidal a la salida del generador Fuente: Elaboración Propia

\section{Acoplamiento electrónico}

El acoplamiento electrónico consiste en los circuitos del control de velocidad del motor y el circuito de recuperación de energía, estos circuitos fueron montados en una base de madera para poder manipularlos más fácilmente como banco de pruebas y brindarles mayor soporte como se muestra en la Figura 12

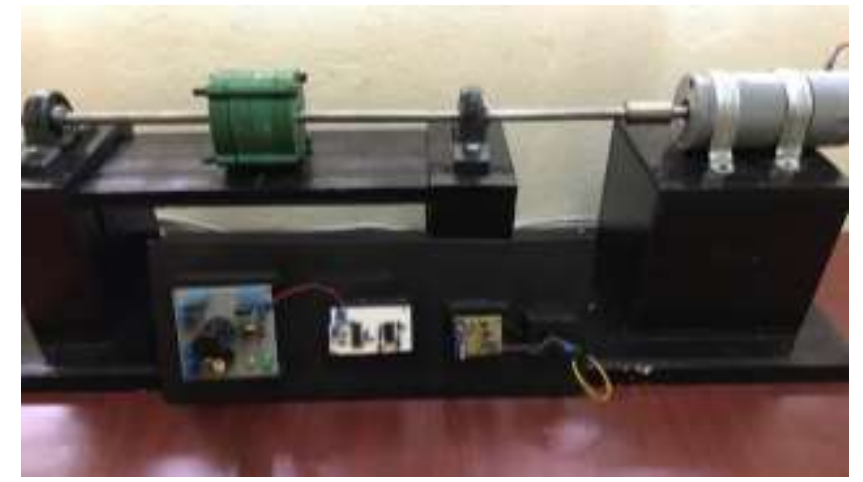

Figura 12 Implementación de circuitos del banco de pruebas

Fuente: Elaboración Propia

\section{Control de velocidad del motor}

En la Figura 13 se muestra el circuito físico implementado en una placa fenólica con tecnología THT, cuyas pistas fueron diseñadas en el software de simulación PROTEUS y se utilizó una construcción modular para la sustitución de los integrados, en caso de deterioro o modificación.

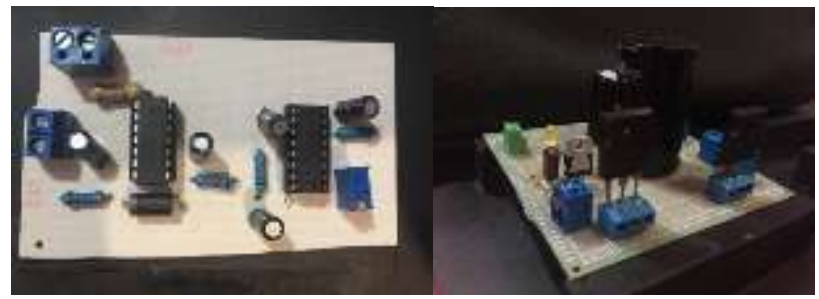

Figura 13 Implementación de circuitos del control de velocidad

Fuente: Elaboración Propia

En la Figura 14 muestra la captura de pantalla del osciloscopio de la señal de control PWM para regular la potencia de salida del control de velocidad, a través del troceador asociado.

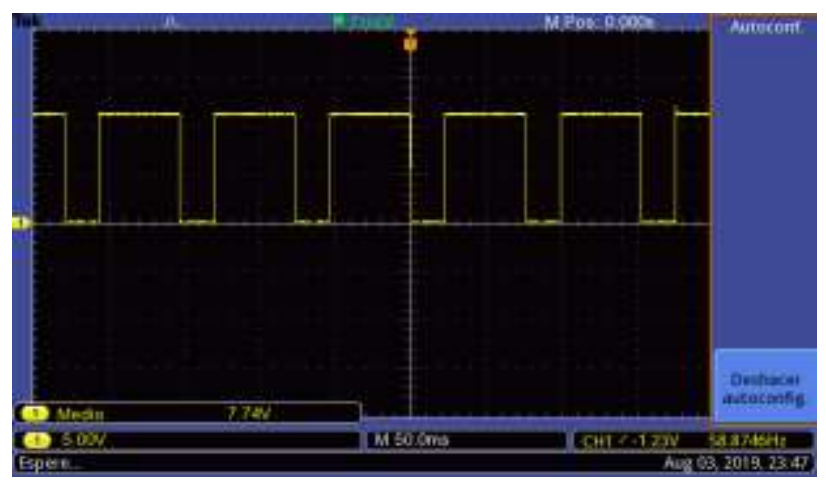

Figura 14 Señal de salida del circuito de control de velocidad

Fuente: Elaboración Propia

\section{Circuito rectificador con filtro y módulos de convertidores Buck y Boost}

En la Figura 15 se muestra la implementación física del circuito rectificador de diodos de germanio con filtro, así como el módulo de convertidor Buck implementados en una placa fenólica con la misma tecnología de construcción THT, así también como la incorporación del módulo integrado comercial de Convertidor Boost Módulo MT3608.

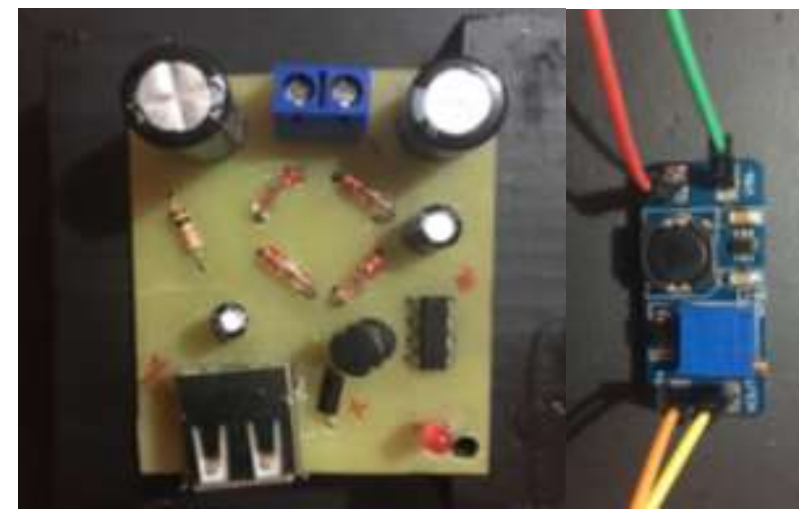

Figura 15 Circuito rectificador, convertidor Buck en placa fenólica y modulo convertidor Boost

Fuente: Elaboración Propia

MASTACHE-MASTACHE, Jorge Edmundo, RAMÍREZ-CHÁVEZ, César León, LOVERA-GARCIA, Jesús Adan y DE LA GARZAMACEDO, José Ángel. Sistema de recuperación de energía basado en emular el movimiento del flujo de agua en tuberías. Revista de Ingeniería Tecnológica. 2020 
Se tomó la captura de pantalla del osciloscopio en la salida del circuito rectificador de diodos de germanio, cuya señal muestra una salida unipolar en $\mathrm{CD}$ con un voltaje pico-pico de $9.8 \mathrm{~V}$ teniendo una pérdida de voltaje relativamente pequeña en la conducción de los diodos. Así mismo se muestra en la siguiente imagen, la señal de salida del rectificador después de la etapa de filtrado. Estas señales se puede observar en la Figura 16, las cuales son la entrada para los módulos de convertidores de potencia CD-CD.

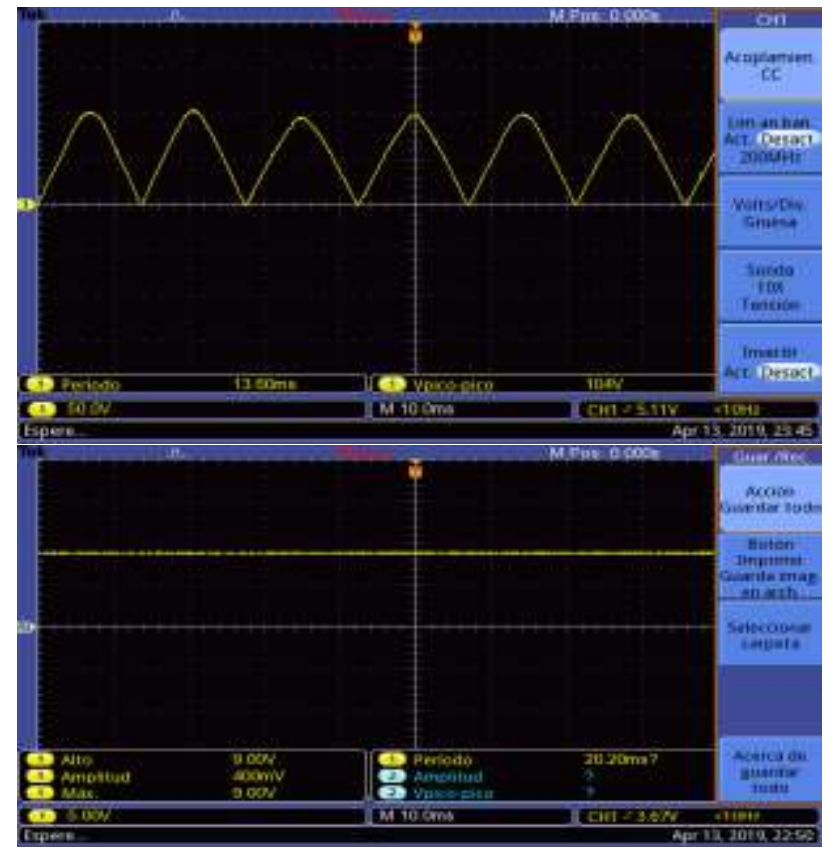

Figura 16 Señal de salida del circuito rectificador sin filtro y con filtro

Fuente: Elaboración Propia

Para reducir y estabilizar el nivel de voltaje, se añadió el convertidor Buck, el cual regula a $5 \mathrm{~V}$ la señal para tener a la salida a una señal más estable, en función a la señal de entrada, la cual es cambiante según la velocidad de giro del sistema mecánico. La Figura 17 muestra la salida en el osciloscopio regulada a $5 \mathrm{~V}$.

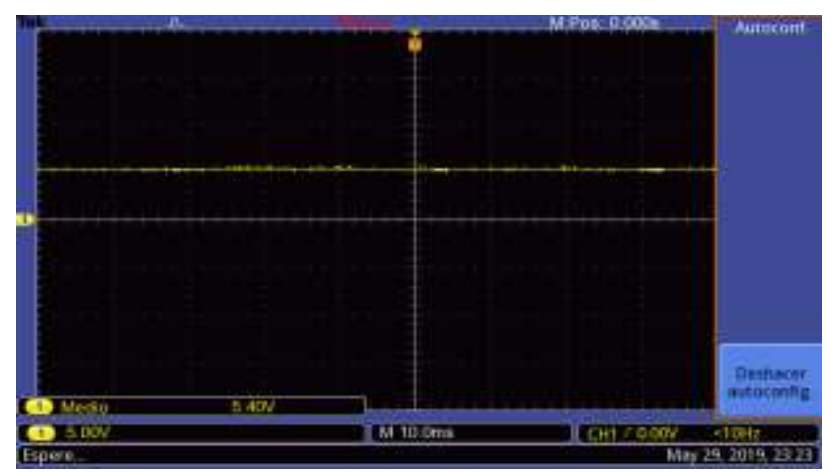

Figura 17 Señal de salida del convertidor Buck.

Fuente: Elaboración Propia
Se añadió al circuito de recuperación de energía un módulo MT3608, que consiste en un convertidor Boost con salida regulable que puede variar de 5 a $28 \mathrm{~V}$ con una corriente máxima de $2 \mathrm{~A}$. Para cumplir con uno de los objetivos de este trabajo como lo es cargar una batería y funcionar como un banco de pruebas de recuperación de energía, en el cual se regula la tensión de salida del voltaje del convertidor Boost hasta obtener $12 \mathrm{~V}$, este nivel de voltaje se muestra en la Figura18 y representa la elevación y adaptación de la potencia para ser utilizado a diferentes cargas.

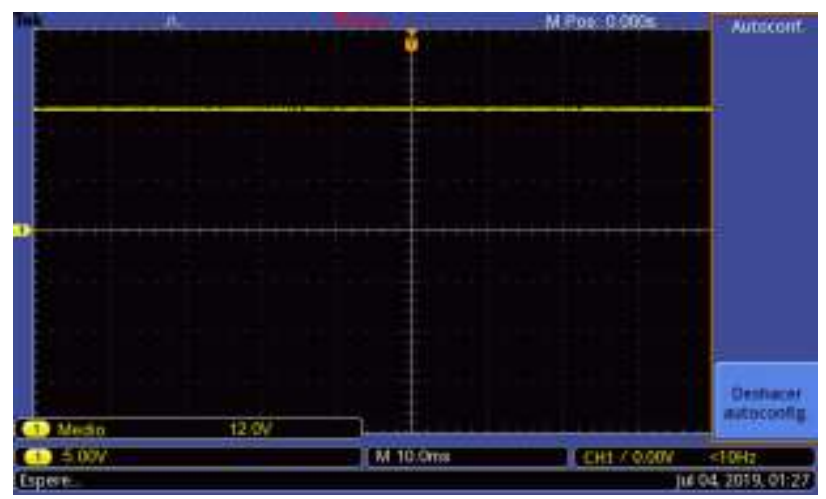

Figura 18 Señal de salida del convertidor Boost a $12 \mathrm{~V}$ Fuente: Elaboración Propia

\section{Agradecimientos}

Los autores de este trabajo agradecemos el apoyo que se ha tenido, para el desarrollo de este proyecto, por parte de la Universidad de Ixtlahuaca y la Escuela Profesional de Ingeniería.

\section{Conclusiones}

El prototipo cumple con objetivo principal de este trabajo, el cual era desarrollar un banco de pruebas para la recuperación de energía eléctrica emulando el flujo de agua en las tuberías de agua potable de las edificaciones a través de un generador de imanes permanentes para el tratamiento de la señal de salida hacia un módulo reductor y elevador de tensión para utilizar esta señal de salida en diferentes aplicaciones.

Este trabajo funge como punto de partida para el análisis de los sistemas mecánicos acoplados a elementos electromecánicos que puedan ser usados para el tratamiento de señales o energías residuales en algún sistema de movimiento. 
Por lo tanto, la principal aportación de este articulo radica en que se realizó una herramienta (banco de pruebas) como base para futuras investigaciones y generaciones de estudiantes dentro del grupo de investigación que podrán desarrollar técnicas de control y electrónica de potencia relacionados con el almacenamiento y tratamiento de energía provenientes de sistemas mecánicos de uso convencional.

\section{Trabajos futuros}

El banco de pruebas contribuye como un fundamento para nuevas líneas de investigación en la Universidad investigaciones relacionadas con la implementación, el control, automatización o la optimización de este prototipo. En cuanto a la construcción y diseño de los componentes mecánicos, las áreas de mejora incluyen una revisión a los componentes de refuerzo del banco de pruebas para garantizar una operación libre de vibraciones. Cabe destacar que este prototipo se puede extrapolar a aplicaciones con flujos de agua más abundantes, por lo que al utilizar la metodología presentada en este trabajo puede adaptarse a diversas aplicaciones.

\section{Referencias}

A. Franco y M. Velázquez, "Una aproximación sociodemográfica al consumo de energía en los hogares mexicanos". México: Consejo Nacional de Población, 2014.

"Encuesta Nacional de Ingresos y Gastos de los Hogares 2014", Instituto Nacional de Estadística y Geografía. México, 2014. [En línea]. Disponible en: https://www.inegi.org.mx/programas/enigh/nc/2 014/. [Accedido: 01-marzo-19].

"Balance Nacional de Energía 2017”, Secretaría de Energía. México, 2017. [En línea]. Disponible en: https://www.gob.mx/cms/uploads/attachment/fi le

/414843/Balance_Nacional_de_Energ_a_2017. pdf. [Accedido: 01-marzo-19].Instruments, N. (s.f.). LabVIEW User Manual.
J. E. Mastache, "Análisis de un Generador Radial de Imanes Permanentes y Propuesta de una Metodología para Optimizar la Eficiencia", Tesis de maestría en Ciencias. Centro Nacional de Investigación y Desarrollo Tecnológico, México, 2016.

M. Rashid, "Electrónica de Potencia. Circuitos, dispositivos y aplicaciones", México: Pearson, 2004. 